\title{
Semi-Equalizing Load in Multi-hop Wireless Networks
}

\author{
B.Abhishek Reddy, Kayiram Kavitha, Ashoka Deepthi Manukonda, R.V.S.Lalitha, \\ N.V.Krishna Rao
}

\begin{abstract}
Scheduling transmissions in a well-organized and fair manner in multi hop wireless network [MWN] is very crucial and challenging .For semi equalizing the load a distributed node scheduling algorithm is used through slot reallocation based on local information swap. The algorithm helps to find the delay or shortest delivery time is achieved when the load is semi-equalized throughout the network. We have simulated the Local voting algorithm and found that the system converges asymptotically toward the optimal schedule. In this paper we propose a congestion free scheme to schedule the node transmissions conflict free. The proposed algorithm achieves better performance than the other distributed algorithms in terms of fairness, average delay, and maximum delay in simulation results.
\end{abstract}

Keywords: Multi-hop wireless networks, node scheduling algorithm, wireless mesh networks, load balancing.

\section{INTRODUCTION}

Modern life is greatly dependent on gadgets ranging from Smart television, Driverless cars, Smart phone etc. These devices require Wireless Networks, Cellular Technology, and routers etc. The Wireless Multi-hop Networks [1] require wireless connectivity in order to disseminate the network functionality. The devices are equipped with a wireless transmitter and receiver to enable communication between the devices and the central base-station as well. The base-station en-route data to the wireless end devices via multiple intermediate nodes. Each such transmission between the wireless devices is termed as a hop. As the data is transmitted by multiple wireless systems before reaching its destined wireless end system, we call such networks as wireless multi-hop networks. With ever growing network traffic, there was much focus on the practical working efficiency of Multi-hop Wireless Networks. In contrast to single wireless links, the multi-hop wireless network can

Revised Manuscript Received on October 15, 2019.

B.Abhishek Reddy, PG Student, Dept. of CSE, Gokaraju Rangaraju Institute of Engineering and Technology, Bachupally, Hyderabad, Telangana, India.

Dr. Kayiram Kavitha, Associate Professor, Dept. of CSE, Gokaraju Rangaraju Institute of Engineering and Technology, Bachupally, Hyderabad, Telangana, India.

Ashoka Deepthi Manukonda, Assistant Professor, Dept. of CSE, Institute of Aeronautical Engineering, Dundigal, Hyderabad, Telangana, India.

Dr. R.V.S.Lalitha, Professor, Dept. of CSE, Aditya College of Engineering, Surampalem, Kakinada, A P, India.

N.V.Krishna Rao, Assistant Professor, Dept. of CSE, Institute of Aeronautical Engineering, Dundigal, Hyderabad, Telangana, India. improve the connectivity and coverage with its intermediate nodes participation in the network. Now-a-days the network coverage and in wireless multi-hop network, the most important requirement is connectivity.

It is more efficient to transmit over short links than the long links. Further they enable better data rate and higher throughput and more efficient use of wireless medium. The major advantage of this wireless medium is to avoid deployment of cables. Thus eliminating the hardware failure issues like cable break, hardware failure, signal disruption, low bandwidth etc.

To improve the operating efficiency of the multi-hop wireless network, efficient channel utilization is highly desired. This leads to node scheduling problem in it. The routing protocols used for the networks like fixed, cellular, and Internet are used for the multi-hop wireless networks for the reasons of performance efficiency. Other networks use unicast, multicast for routing whereas the multi-hop wireless networks employ multiple channels for routing. Also, multiple paths are created for its data transmission. Thus, the nodes need to follow a schedule. Node scheduling [2] is to schedule the transmission chance to a set of nodes without common obstruction among the transmitting nodes.

Several algorithms are available in the literature, for node scheduling in multi-hop wireless networks like DRAND [3], Load-Based Transmission Scheduling (LoBaTS) [5], LQF algorithm[6], Lyui's algorithm [4],

In this paper, we discuss the issue of node scheduling in multi-hop wireless networks. Every transmission chance is scheduled to many nodes with guarantee of no shared impedance among any transmitting nodes. More explicitly, two nodes can be scheduled on a similar availability (and transmit at the same time). Hence, they should not interfere with each other. So, we present a congestion free scheme to schedule the node transmissions conflict free.

We present the detailed Literature Survey in the next Section.

\section{LITERATURE SURVEY}

The authors in [3] proposed Distributed Randomized TDMA Scheduling for Wireless Ad Hoc Networks (DRAND), which is the first fully distributed version of RAND. The algorithm is viable in adjusting to nearby topology changes without bringing about global overhead in the planning and time synchronization is not required. Because of these features, frequency or code scheduling are some of the scheduling problem used by DRAND in wireless network.

Lyui [4], is used for packet radio networks to assign the collision-free broadcast

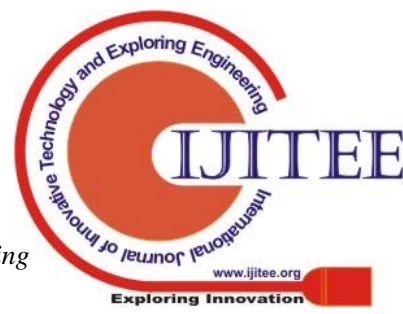


transmissions. Contention-based channel-access protocol is not required and it is distributed and fair. The assignment algorithm of Lyui's properties particularly used for dynamic ad hoc networks.

The work in [5] deals with Load-Based Transmission Scheduling (LoBaTS) protocol for wireless ad hoc networks. In this network the terminals are required to forward unequal amounts of traffic so for that the transmission scheduling protocol provides equal transmission capacity to terminals which are in the network which may result in traffic congestion as load in the traffic increases. The LoBaTS protocol allows terminals to alter their existing transmission schedules so that those terminals which are required to forward more traffic can reserve additional transmission slots, thereby alleviating traffic bottlenecks.

An effective and reasonable hub planning is a major challenge in multi-jump remote networks. A conveyed hub booking algorithm, called Local Voting [7]. The thought originates from finding that the delay or shortest delivery time is gotten when the heap is stabled all through the network.fairness Average delay, maximum delay by local voting has exhibited better performance when compared to other scheduling algorithms from the literature. However we find some shortcomings in this scheme. When the nodes are following a schedule, we found that the participants in the queue are waiting for a longtime. Hence there is unnecessary delay in the network. So, we propose a conflict free scheme for efficient transmission in the next Section.

\section{IMPLEMENTATION METHODOLOGY}

In the node scheduling problem, the mutual interference should not present when set of nodes is assigned with transmission opportunity among any transmitting nodes. Under node scheduling, if two nodes do not have common neighbors then they are assigned to same time slot (and transmit simultaneously). We propose a congestion free node scheduling algorithm. The idea behind the algorithm is to minimize the delivery time in a network was originated by its observation, if the ratio of the queue length over the number of allocated slots is semi-equalized throughout the network. We call this ratio the load of each node. The proposed algorithm will allow the nearby nodes to exchange slots such that they ultimately semi-equalizes [8] the load in the network. The relation between its neighbors and load of each node is to determine the no of slots that are exchanged; certain slot exchanges are not possible due to interference with other nodes. We likewise show that its reenactment is exceptionally near a unified algorithm. The displayed algorithm is a change of the Local Voting convention with non-disappearing to zero stage size. It has a place with the more broad class of stochastic estimate decentralized algorithms with diminishing to zero stage size.

In multi-hop communication, two nodes communicate using single transmission slot, without collision. But, both the nodes should not have any neighbors in commonelse, a collision may occur. In general collision results in data loss. Efficient node scheduling means to avoid collisions from happening. Hence nodes need to be scheduled for collision free network. Every node maintains a queue in FCFS basis for all its outgoing packets. The queue length is treated to be limitless as the packets are all generated dynamically. Each node has their own timeline and corresponding data frames to be sent. So two neighbors may exchange their free slots for their mutual convenience too. Hence the slot exchanges are possible and is termed as slot re-assignment problem [9].

The primary goal in a multi-hop network is to maintain efficient data flow without loss and collisions [10].We measure the throughput and end-to-end delay per packet of each flow to prove the efficiency in channel utilization. All these network parameters are measure per node instead of the entire network. The proposed scheme considers only the slot reassignment problem.

The ideal requirement for an efficient network is not to allocate slots to empty queue. This is done at the beginning of every frame to ensure that none of the slots go waste without any frames. So, nodes release all the reserved slots as soon as they find an empty queue. Also, the neighborhood nodes should not have free time slots to be maximal if a node has a positive queue. All free slots are allocated to the nodes that have a non-empty queue. When there is a race between two nodes, the node with less load is preferred to use the channel in contrast to the node with higher load (queue length).

Another objective is to keep the network balanced. This is conceived by semi-equalizing the load in the entire network. Here load refers to the ratio of queue length over the number of allocated slots. Ideally the number of slots per node should be able to drive the backlogged traffic to its destination without delay. In this regard, two neighboring nodes can exchange their free slots for optimal channel utilization.

We present our contention free algorithm to accomplish the shortest start to finish delivery time and most prominent fairness contrasted with other disseminated algorithms for various network densities. The exhibited algorithm is a change of the Local Voting convention with nonevaporating to zero stage size.

In our algorithm, we perform hub planning by utilizing the spaces without information about the individual streams. From the time when multi-bounce start to finish delay is the entirety of nodal delays on the start to finish way, we anticipate that our algorithm should convey additionally great multi-jump start to finish delay performance.

\section{Algorithm1: Pseudocode of Conflict free Scheduling} set transfer 0

proc schedule $\{$ alt_ftp alt_ftp1 alternate $\}\{$ global ns globalftp_list globaltcp_list global transfer globalmhop set now [\$ns now] set $\mathrm{j} 0$ foreach item \$tcp_list \{ set queue [\$item set cwnd_] if $\{$ \$queue $>20 \& \&$ \$transfer $==0\}\{$ set $\mathrm{k} 0$

foreach ftp \$ftp_list \{ if $\{\$ \mathrm{k}==\$ \mathrm{j}\}\{$ \$ns at \$now "\$ftp

stop" 
if $\{\$$ transfer $==0\}\{$

set hop [lindex \$mhop \$j]

puts "InTraffic congested at hop\$hop and using alternate path hop\$alternateln"

\$ns at \$now "\$alt_ftp start"

\$ns at \$now "\$alt_ftp1 start"

set transfer 1

\author{
\} \\ \} \\ set $\mathrm{k}[\operatorname{expr} \$ \mathrm{k}+1]$ \\ \} \\ \} \\ set $\mathrm{j}[\operatorname{expr} \$ \mathrm{j}+1]$ \\ \} \\ set time [expr \$now + 1.0] \\ \$ns at \$time "schedule \$alt_ftp \$alt_ftp1 \$alternate" \\ \}
}

The proposed Conflict-free algorithm comprises of two capacities: mentioning and discharging leisure time spaces, and burden balancing. For the primary capacity nodes are inspected successively toward the start of each casing. On the off chance that a node has a vacant line, at that point it discharges all its time openings. In the event that a node has a positive excess (for example its line isn't unfilled), at that point it is given time spaces. Unsurpassed openings are inspected successively, and the most readily accessible time spaces that are discovered, which are not held by onebounce or two-jump neighbors for transmission, are distributed to the node. In the event that no accessible opening is discovered, at that point no new opening is dispensed to the node. Despite what might be expected, if the line of the node is seen as vacant and the node has designated spaces, at that point all openings are discharged.

We have experimented the local voting algorithm and our Conflict free algorithm to demonstrate the performance analysis in the next Section.

\section{PERFORMANCE ANALYSIS \& RESULTS}

The planning algorithm evaluation is being engaged by the reproduction instrument. The appraisal happens dependent on two situations. Every association will be begun with a precise number of bundles when a variable no of associations are considered. This speaks to the reaction to an unexpected burst of traffic. Number of associations is being changed with the end goal that the various loads in the network are adjusted. The reproduction will be executed till the entire bundles arrive at the goal in the primary situation. Associations are included continually, following a Poisson procedure. While changing the association appearance rate the heap is being aligned in the second situation. This situation is executed for fixed time span.

The deliberate measurements for every association are:

- The delivery time is where every one of the bundles of an association will arrive at the last goal ;

- The delay is where the goal gets the parcel from a source at a specific minute

- The throughput is where the parcels in the association are being partitioned utilizing an opening or a specific time distinction between the source and goal.

The simulation software is categorized in to 4 packages: In network package algorithms and network elements are being implemented, in stability package the different scenarios are being executed , and in simulator package discrete-event simulator is being implemented using the objects which are present, the application package the network connections and the statistics gathering functionality is being implemented. Some of the network functions that were implemented in the simulation tool include the following: the application layer is represented by a connection object. For the purposes of this simulation, random source and destination contains in every connection object. The no of packets are being initialized which are to be transmitted. In the first scenario, 100 packets are present in each connection. The slot reservation is represented by the reservation object. The nodes which are blocked due to the reservation and fields for transmitting nodes are present in the reservation object. The network functions are implemented by the Network object, such as routing.

For every simulation, random source and destination nodes are generated by 1 to 30 simultaneous connections. For every 5 time units 1 packet is generated by each connection till the total no of packets per connection is being generated.

Conflict-free algorithm gets the better performance then the local voting algorithm in shortest delivery time, fixed no of slots to every node is assigned to the above algorithms without considering the traffic conditions.

With the fairness index proposed by Jain, the fairness in terms of throughput among connections is being calculated as shown in figure (a). The conflict-free algorithm gets better fairness than other algorithm, despite the quantity of simultaneous associations.

The average end-to-end delay is being calculated as shown in fig (b)., the conflict-free algorithm is achieves a better performance than the Local voting algorithm.

The Throughput is being calculated as shown in figure (d) where the packets in the connection are being divided using a slot or a particular time difference between the source and destination

The maximum end to end delivery time is being calculated as shown in fig (d) which is an important metric. It is identified with the limit of the network. However, the act of the conflict-free algorithm is near ideal.

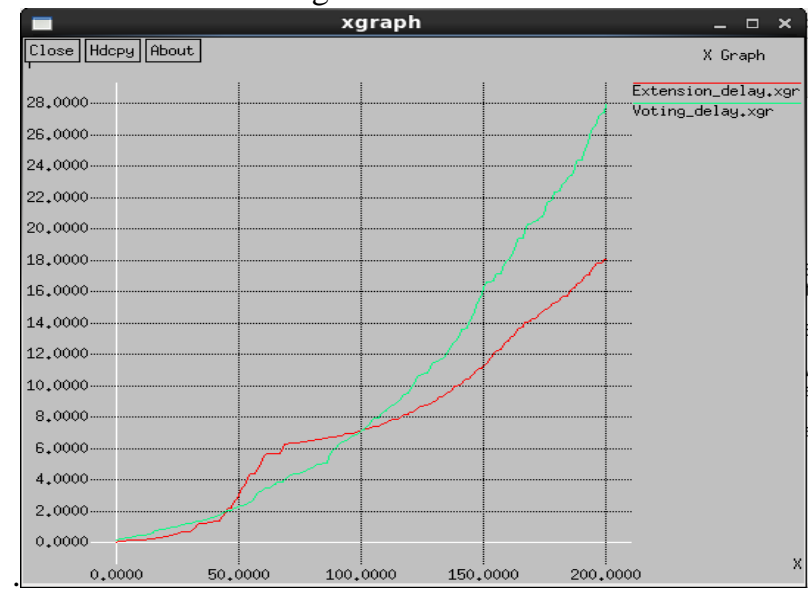

Figure a. Graph showing delay in the local voting and conflict free algorithms. 


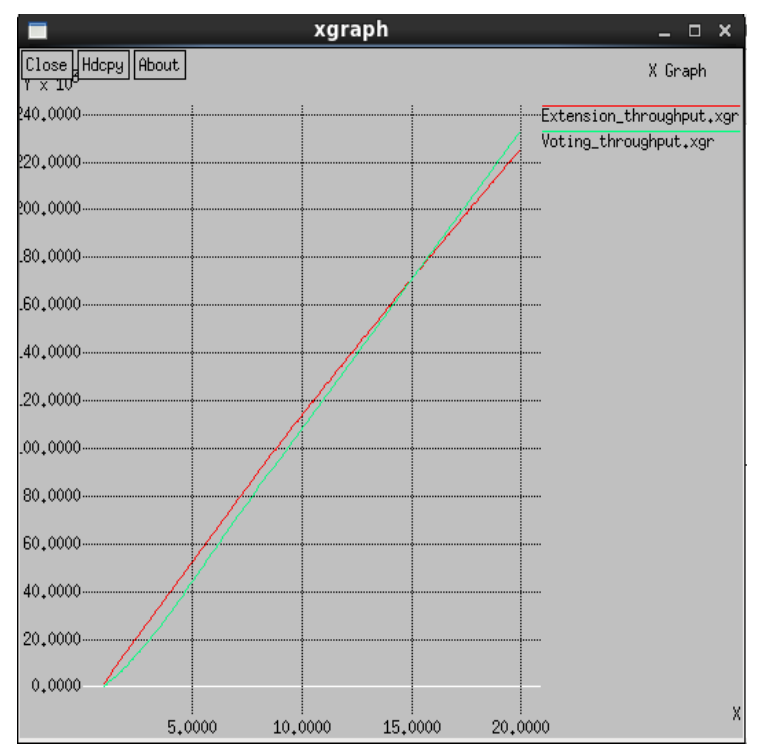

Figure b. Graph showing throughput in the local voting and conflict free algorithms.

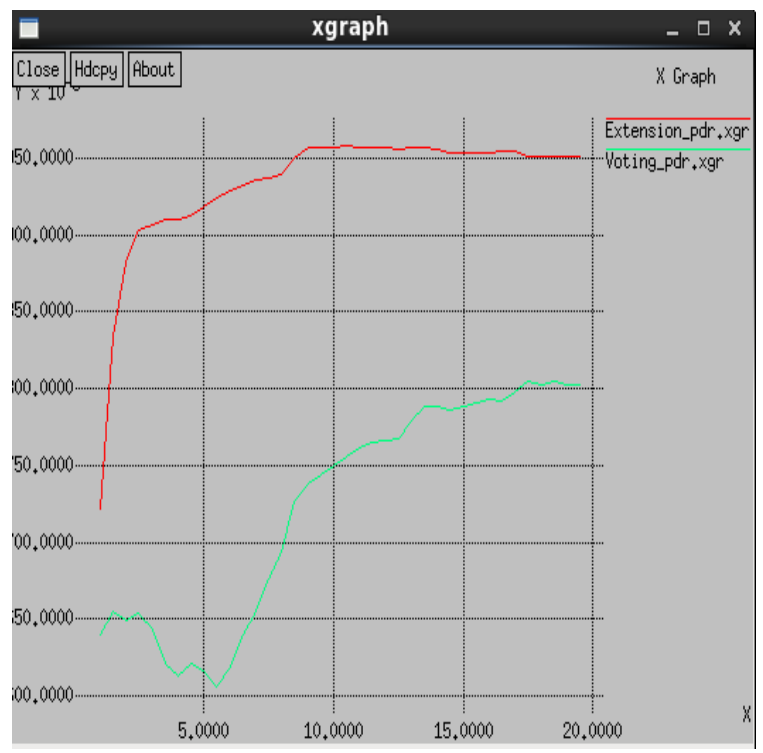

Figure c. Graph showing packet delivery ratio in the local voting and conflict free algorithms.

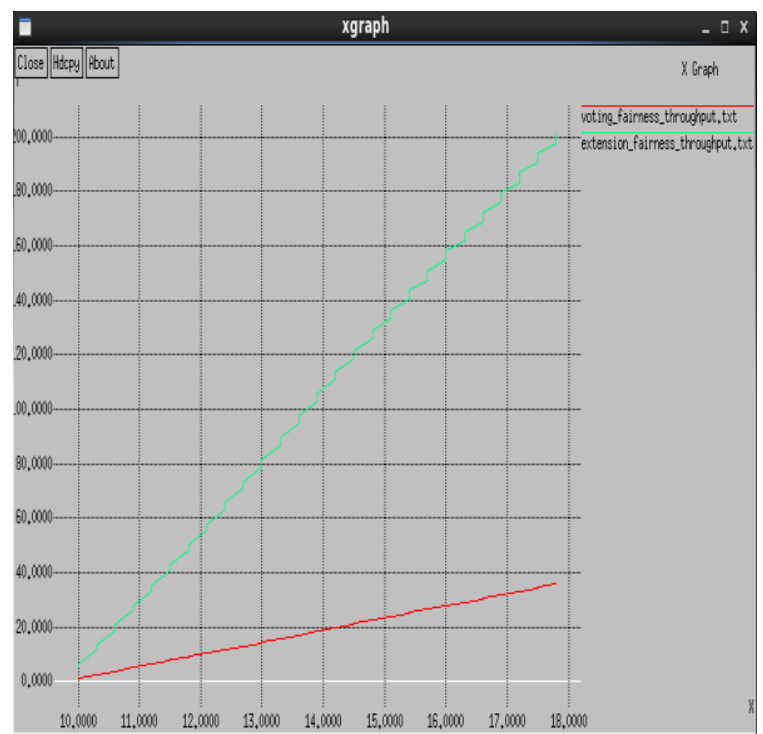

Figure d.Graph showing fairness in the local voting and conflict free algorithms.

\section{CONCLUSION}

The issue of planning is one of the enormous difficulties in multi-jump remote networks. In this paper we examined the association of planning and burden adjusting with a objective to semi-equalize the division of the quantity of openings designated to every node over the line length of the node. The issue of remote booking is portrayed as a heap adjusting issue. At long last, we found the conditions that ought to be met all together for the without conflict algorithm to accomplish estimated accord, and therefore upgrade the delivery time all through the network. Simulation results approved the theoretical investigation and demonstrated that the delivery times are limited with the utilization of the Conflict-free algorithm. The conflict-free algorithm accomplishes preferred performance over the distributed algorithms which are known from the writing as far as fairness, the greatest delay, the normal delay. To condense, we indicated the upside of burden adjusting when performing planning for remote multi-jump networks, proposed Conflict-free algorithm for burden adjusting/booking.

\section{REFERENCES}

1. C. Bettstetter and C. Hartmann, "Connectivity of wireless multihop networks in a shadow fading environment," Wireless Netw., vol. 11, no. 5, pp. 571-579, 2005

2. Tian, Di, and Nicolas D. Georganas. "A coveragepreserving node scheduling scheme for large wireless sensor networks." In Proceedings of the 1st ACM international workshop on Wireless sensor networks and applications, pp. 32-41. ACM, 2002.

3. Rhee, Injong, Ajit Warrier, Jeongki Min, and Lisong Xu. "DRAND: Distributed randomized TDMA scheduling for wireless ad hoc networks." IEEE Transactions on Mobile Computing 8, no. 10 (2009): 1384-1396.

4. Hammond, Joseph L., and Harlan B. Russell. "Properties of a transmission assignment algorithm for multiple-hop packet radio networks." IEEE Transactions on Wireless Communications 3, no. 4 (2004): 1048-1052.

5. B. J. Wolf, J. L. Hammond, and H. B. Russell, "A distributed load-based transmission scheduling protocol for wireless ad hoc networks," in Proc.of Int. Conf. on Wireless Comm. and Mobile Comp., 2006, pp. 437-442.

6. Dimakis and J. Walrand, "Sufficient conditions for stability of longest-queue-first scheduling: Second-order properties using fluid limits," Ad-vances in Applied probability, pp. 505-521, 2006.orks," in Proc.of Int. Conf. on Wireless Comm. and Mobile Comp., 2006, pp. 437-442.

7. Vergados, Dimitrios J., Natalia Amelina, Yuming Jiang, Katina Kralevska, and Oleg Granichin. "Toward optimal distributed node scheduling in a multihop wireless network through local voting." IEEE Transactions on Wireless Communications 17, no. 1 (2017): 400-414.

8. C.-T. Chiang, H.-C. Chen, W.-H. Liao, and K.-P. Shih, "A decentralized minislot scheduling protocol (DMSP) in TDMA-based wireless mesh networks," J. Netw. Comput. Appl., vol. 37, pp. 206-215, Jan. 2014

9. Kayiram Kavitha, Polsani Rajashree Rao, Sreeja Tummala, Gajarla Vasavi, and Dr.R.Gururaj, Dual Tree Data Routing Scheme for Wireless Sensor Networks, Third International Conference on Advances in Information

Technology and Mobile Communication, AIM - 
2013, 26-27, April 2013, Bangalore, India.

10. Kayiram Kavitha, Vinod Pachipulusu, Sreeja Thummala, R.Gururaj. Article: Energy Efficient Query Processing for WSN based on Data Caching and Query Containment, in International Journal of Computer Applications, Vol. 89, no. 19, Page no. 4-8, March 2014, Published by Foundation of Computer Science, New York, USA.

11. Sharma D, Kavitha K, Gururaj R. Article: Estimating Node Density for Redundant Sensors in Wireless Sensor Network, in International Journal of Sensor Networks and Data Communications, Vol.4, Issue 2 no.127, November 2015, Published by OMICS International.

12. R.V.S.Lalitha, K.Kavitha, N.V.Krishna Rao, G.Rama Mounika, V.Sandhya, Smart surveillance using Smart doorbell, International Journal of Innovative Technology and Exploring Engineering, Volume-8, Issue-8 June, 2019.

\section{AUTHOR PROFILE}

B.Abhishek Reddy is pursuing M.Tech in Gokaraju Rangaraju Institute of Engineeringand Technology, Bachupally, Hyderabad, Telangana, India

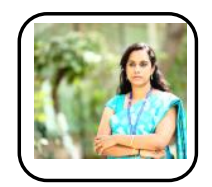

Dr.Kayiram Kavitha is currently working as Associate Professor in the Department of CSE, Gokaraju Rangaraju Institute of Engineeringand Technology, Bachupally, Hyderabad, Telangana, India. Her areas of interest include Wireless Sensor Networks, Security in Internet Of Things, Web Services and Mobile Computing

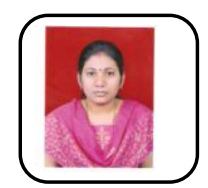

Ashoka Deepthi Manukonda, Assistant Professor, Department of CSE , Institute of Aeronautical Engineering, Dundigal, Hyderabad, Telangana, India.

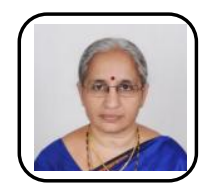

Dr. R V. S. Lalitha is currently working as Professor in the Department of CSE, Aditya College of Engineering and Technology, Surampalem, Kakinada., AP, India. Her areas of interest include Vehicular Ad hoc Networks, Data mining, and Mobile computing.

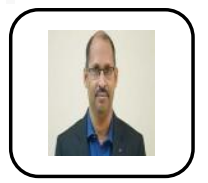

N V Krishna Rao, Assistant Professor, Department of CSE , Institute of Aeronautical Engineering, Dundigal, Hyderabad, Telangana, India. 